\title{
Interaction and imitation in a world of Quixotes and Sanchos
}

\author{
Francisco Cabo · Ana García-González
}

the date of receipt and acceptance should be inserted later

\begin{abstract}
This paper studies a two-population evolutionary game in a new setting in between a symmetric and an asymmetric evolutionary model. It distinguishes two types of agents: Sanchos, whose payoffs are defined by a prisoner's dilemma game, and Quixotes, whose payoffs are defined by a snowdrift game. Considering an imitative revision protocol, a revising agent is paired with someone from his own population or the other population. When matched, they observe payoffs, but not identities. Thus, agents in one population interact and imitate agents from their own population and from the other population. In this setting we prove that a unique mixed-strategy asymptotically stable fixed point of the evolutionary dynamics exists. Taking as an example the compliance with social norms, and depending on the parameters, two type of equilibrium are possible, one with full compliance among Quixotes and partial compliance among Sanchos, or another with partial compliance among Quixotes and defection among Sanchos. In the former type, Sanchos comply above their Nash equilibrium (as they imitate compliant Quixotes). In the latter type, Quixotes comply below their Nash equilibrium (as they imitate defecting Sanchos).
\end{abstract}

Keywords Two-population evolutionary game, heterogeneous preferences, symmetric and asymmetric games.

JEL Codes: C73, D01, Q58.

IMUVa, Universidad de Valladolid. Avda. Valle Esgueva, 6, 47011, Valladolid, Spain. Corresponding author: Francisco Cabo. Tel: +34 983 186662. E-mail: pcabo@eco.uva.es. ORCID: Francisco Cabo (0000-0003-2958-9595); Ana García-González (0000-0002-2670-9574) 


\section{Introduction}

In symmetric evolutionary games, it is well established that an evolutionary stable strategy (ESS) is a Nash equilibrium isolated in the set of symmetric Nash equilibria. When turning to asymmetric games, Selten (1980) showed that every ESS must be a pure Nash equilibrium. Strictly mixed-strategy Nash equilibria are not asymptotically stable (as proven in Hofbauer and Sigmund 1998, under the replicator dynamics, or in Samuelson and Zhang 1992, under a more general setting). Consequently, each type of player must use one of the pure strategies. This is a somewhat unsatisfactory result since many games do not have an equilibrium in pure strategies, and so an ESS does not exist for such games. ${ }^{1}$ As commented by Samuelson and Zang (1992) much of the literature on equilibrium refinement is based on the assumption that there are interesting equilibria which are not strict.

This paper proposes a new setting in between a symmetric evolutionary model and an asymmetric one. Two populations of individuals are considered. Players from the two populations share the same strategy set and interact among each other. The game differs from a symmetric game in which all Sanchos would only interact among each other but not with Quixotes (and vice versa). Also the current model differs from the classical asymmetric game ${ }^{2}$ where Sanchos would interact with Quixotes but not with Sanchos (and vice versa). More importantly, when a player receives a revision opportunity, he can compare his payoff by random matching with individuals from his same population, but also with individuals from the other population. Under this specification, there exists a unique mixed-strategy asymptotically stable equilibrium. In particular, in one population agents follow a pure strategy, while agents in the other population play mixed strategies.

To illustrate this game, we assume two types of individuals confronted with a social dilemma, such as the compliance with social norms. Dawes (1975) defines a social dilemma as a situation in which: "(1) each person has available a dominating strategy, and (2) the collective choice of dominating strategies results in a deficient outcome". Later on, Liebrand (1983) stated that: “Dawes's requirement of a dominating strategy for each person does not appear to be crucial for considering a situation a social dilemma. What is critical is that a strategy can be chosen that ultimately results in an outcome that is deficient for all persons involved, and that nonetheless can be attractive since in some circumstances that strategy yields the best payoff for the person choosing it". We assume that the first group of individuals, standard

\footnotetext{
1 This is particularly so for non-trivial extensive form games, as stated by Mailath (1998).

2 Oprea et al. (2011) study a two-population snowdrift game in which row players play only column players and vice versa. Other interesting asymmetric games are, for example, Antoci et al. (2009) and (2012), in finance and environmental problems, or Antoci et al. (2011), in traffic congestion.
} 
economic agents or Sanchos, perceive the social dilemma as a prisoner's dilemma (the most widely used paradigm to study human cooperation). In the other population, Quixotes experience a higher utility from compliance and a stronger disutility from defection. When the opponent complies, defection is still preferred, but if the opponent defects, then compliance is the best strategy. Thus, for Quixotes, the compliance dilemma is relaxed, and is described as a snowdrift or chicken game (see, for example, Doebeli et al. 2004).

For a one-shot prisoner's dilemma game, mutual defection is the only Nash equilibrium. By contrast, the snowdrift game presents a Nash equilibrium in mixed strategies (and two asymmetric pure Nash equilibria). Correspondingly, for a single population evolutionary game, the share of compliant agents in a prisoner's dilemma game vanishes at the equilibrium, while it converges towards a mixed strategy at which some individuals defect while others comply, in the snowdrift game.

The novelty of our two-population evolutionary game is twofold. It considers that agents in one population play a game against agents in both populations (interpopulation interaction). Moreover, when a player receives a revision opportunity he can be matched with an agent within his own population (as it is standard in multipopulation games ${ }^{3}$ ), but can also be matched with an agent in the other population. When matched, they exchange information about each other's payoff, but they do not acknowledge whether the opponent is of the same type or belongs to the other population. The basis for inter-population imitation is to assume that an agent knows his payoff (associated with his current strategy), while also learning the strategy of another agent with whom he is randomly paired. Moreover, he gets imperfect information about the payoff to this agent. Assuming they play different strategies, the more dissimilar the payoffs, the more likely the agents are to perceive this dissimilarity, and the greater the probability that the player with lower payoff changes his strategy. Thus, individuals belonging to one population interact with, and imitate, agents within their own population as well as individuals in the opposite population.

In our setting, we characterize the condition under which there always exists a unique mixed-strategy asymptotically stable fixed point of the evolutionary dynamics. This equilibrium can be of two types. Firstly, if Quixotes are considerably different from Sanchos, especially when there is a larger payoff associated with compliance rather than a stronger penalty to defection, then all Quixotes comply together with some Sanchos. A pure strategy (compliance) is played in the population of

3 See, for example, Sandholm (2010). The mean dynamic for a multi-population game is presented in Chapter 4, page 124, for the general case, and in Chapter 5, page 153, for the imitative dynamics. In the standard setting, the evolutionary dynamics in one population depends on the payoffs matrix within this specific population and the compliance rate in all populations. 
Quixotes, while a mixed-strategy equilibrium characterizes the population of Sanchos. Secondly, under the alternative condition, Quixotes partially comply, while Sanchos never comply. Hence, again one population is characterized by a pure strategy (defection) and the other population is characterized by mixed strategies. Although the two equilibria are asymptotically stable, they are not necessarily Nash equilibria. This occurs because the inter-population imitation leads some individuals in one population to "mistakenly" imitate agents from the other population, even though it is not necessarily in their best interest. In the first type of equilibrium Sanchos comply above their Nash equilibrium as they imitate the compliant behavior of Quixotes. Correspondingly, in the second type, Quixotes comply below their Nash equilibrium because they imitate the defecting behavior of Sanchos.

The rest of the paper is organized as follows. Section 2 presents the two-population evolutionary game, assuming inter-population interaction and imitation. It studies two examples introducing the two alternative equilibria. Section 3 describes the general model, characterizes the two type of equilibrium and proves their asymptotic stability. Furthermore, it compares these stable equilibria to the unstable Nash equilibrium. Section 4 provides conclusions. Proofs can be found in the Appendix.

\section{Inter-population interaction and imitation: a two-population game}

This section describes a two-population evolutionary game involving two type of individuals: Sanchos and Quixotes. The two populations have the same strategy set, and they differ on their preferences or payoff matrices. As an example, we consider a collective action problem such as the compliance with social norms. Each player is better off if all players follow the rules, although there is an incentive to defect and enjoy the benefits provided by the compliance of others.

For the population of Sanchos, the collective action problem is represented in the canonical way as a prisoner's dilemma game, where players decide between compliance and defection. For example, consider the following the payoff matrix:

\begin{tabular}{c|c|c} 
& $\mathrm{C}$ & $\mathrm{D}$ \\
\hline $\mathrm{C}$ & $(10,10)$ & $(0,20)$ \\
$\mathrm{D}$ & $(20,0)$ & $(1,1)$ \\
\hline
\end{tabular}

These agents have an incentive to defect, regardless of what the opponent chooses. Under this prisoner's dilemma specification, compliance is dominated by defection and the unique Nash equilibrium is mutual defection.

In addition to this type of agent, we also take into account a second population, named Quixotes, with different payoffs. Compared to Sanchos, these individuals re- 
ceive a greater payoff for compliance (7 more units) and a lower payoff for defection (2 less units), with the following payoff matrix:

\begin{tabular}{c|c|c} 
& $\mathrm{C}$ & $\mathrm{D}$ \\
\hline $\mathrm{C}$ & $(17,17)$ & $(7,18)$ \\
$\mathrm{D}$ & $(18,7)$ & $(-1,-1)$ \\
\hline
\end{tabular}

This matrix still represents a social dilemma, because Quixotes still have an incentive to defect when others comply $(18>17)$. Nonetheless, the main characteristic that differentiates Quixotes from Sanchos is that the former prefer to be the sole individual who complies over mutual defection $(7>-1)$. Therefore, the problem for these players is not characterized by the prisoner's dilemma, but by a snowdrift or chicken game. This game has two asymmetric pure Nash equilibria, $(1,0),(0,1)$ and one symmetric mixed strategy Nash equilibrium ${ }^{4}(\Delta, 1-\Delta)=(8 / 9,1 / 9)$.

A population game defined exclusively for the population of Sanchos would be characterized by the convergence towards an ESS of zero compliance. Similarly, for a population of Quixotes, the compliance rate at the equilibrium would be $\Delta$.

To define the two-population game, we assume a unit mass population divided between Sanchos and Quixotes. For simplicity we assume a 1/2 mass of Sanchos and an equal $1 / 2$ mass of Quixotes. Denoting by $p$ the ratio of agents in the population of Sanchos who comply, and by $q$ the ratio of compliant Quixotes, the set of social states can be written as $X=\{\mathbf{x}=(p, 1-p, q, 1-q) / 2: p \in[0,1], q \in[0,1]\}$. For each social state, the payoff function for the two-population game can be defined as a function ${ }^{5}$ of the state $\mathbf{x}$, or simply as a function of the share of the total population who complies (adding up Sanchos and Quixotes), $y=(p+q) / 2$ :

$$
\frac{1}{2}\left(\begin{array}{cccc}
10 & 0 & 10 & 0 \\
20 & 1 & 20 & 1 \\
17 & 7 & 17 & 7 \\
18 & -1 & 18 & -1
\end{array}\right)\left(\begin{array}{c}
p \\
1-p \\
q \\
1-q
\end{array}\right)=\left(\begin{array}{c}
10 y \\
19 y+1 \\
10 y+7 \\
19 y-1
\end{array}\right) \equiv\left(\begin{array}{c}
\pi_{\mathrm{c}}^{\mathrm{s}}(y) \\
\pi_{\mathrm{D}}^{\mathrm{s}}(y) \\
\pi_{\mathrm{c}}^{\mathrm{Q}}(y) \\
\pi_{\mathrm{D}}^{\mathrm{Q}}(y)
\end{array}\right)=\pi(y) .
$$

This share determines the payoff of an agent in population $h \in\{S, Q\}$ playing strategy $i \in\{C, D\}$, denoted as $\pi_{i}^{h}(y)$. The $4 \times 4$ matrix in (1) shows that individuals in one population interact with agents from their own population and agents from the other population. A standard symmetric two-population evolutionary game would be characterized by a block diagonal matrix. Correspondingly, an asymmetric game would be associated with a matrix whose main diagonal blocks are zero matrices.

\footnotetext{
${ }^{4}$ At this equilibrium, the expected payoff associated with compliance equates the expected payoff associated with defection.

${ }^{5}$ Superscripts S and Q refer to Sanchos and Quixotes, and subscripts C and D refer to compliance and defection.
} 
From expression (1) it follows that Quixotes get 7 more units from compliance and 2 less units from defection than Sanchos, regardless of the social state, i.e. regardless of the compliance rate in the total population, $y$. Moreover, the payoff function in (1) clearly states that $\pi_{\mathrm{D}}^{\mathrm{s}}(y)-\pi_{\mathrm{c}}^{\mathrm{s}}(y)=9 y+1>0, \forall q \in[0,1]$, and therefore defection always dominates compliance in the population of Sanchos. For Quixotes, $\pi_{\mathrm{D}}^{\mathrm{Q}}(y)-\pi_{\mathrm{C}}^{\mathrm{Q}}(y)=9 y-8$ and only a large compliance rate in the overall population, $y>8 / 9$, makes defection more rewarding than compliance. By contrast, as more individuals defect ( $y$ decays below 8/9), the incentive to defect is outweighed by the damage associated with mutual defection. The threshold is the Nash equilibrium in mixed strategies if only a single population of Quixotes existed: $\pi_{\mathrm{C}}^{\mathrm{Q}}(y) \gtrless \pi_{\mathrm{D}}^{\mathrm{Q}}(y)$ if and only if $y \lessgtr \Delta \equiv 8 / 9$.

Next we specify a dynamic model which explains how each agent reconsiders his strategy choice. We will consider an imitative revision protocol as the updating procedure, specifically, pairwise proportional imitation. An interesting property of the imitative dynamics is that it admits rest points that are not Nash equilibria. ${ }^{6}$ More importantly, the particular setting proposed will make a mixed-strategy asymptotically stable rest point possible. At the equilibrium, agents do not necessarily play pure strategies. Moreover, this equilibrium is not a Nash equilibrium.

An imitative revision protocol: Pairwise proportional imitation:

The dynamics governing the evolution of the compliance rates among Sanchos and Quixotes stem from an imitative revision protocol. The dynamics of the compliance rate for Sanchos, $\dot{p}$, is equal to the share of defecting Sanchos, $1-p$, times the probability that a defecting Sancho switches to compliance, $\rho_{\mathrm{DC}}^{\mathrm{s}}$, minus the share of compliant Sanchos, $p$, times the probability that a compliant Sancho switches to defection, $\rho_{\mathrm{CD}}^{\mathrm{s}}$ :

$$
\dot{p}=(1-p) \rho_{\mathrm{DC}}^{\mathrm{s}}-p \rho_{\mathrm{CD}}^{\mathrm{s}} .
$$

Likewise, the dynamics of the ratio of compliant Quixotes can be written as

$$
\dot{q}=(1-q) \rho_{\mathrm{DC}}^{\mathrm{Q}}-q \rho_{\mathrm{CD}}^{\mathrm{Q}}
$$

The two-population game analyzed here differs from the standard formulation in the sense that players in one population interact with and also imitate players of their own kind but also players in the other population. Inter-population interaction and imitation is feasible based on three assumptions. First, the two populations involved have the same strategy set: compliance and defection $\{C, D\}$ (only with different

\footnotetext{
6 See Sandholm (2010), Chapter 5, subsection 5.4.6, page 164. As examples, the state at which all Quixotes and all Sanchos comply, or the state at which no agent in either population complies are rest points and not Nash equilibria.
} 
payoffs). Second, individuals think that they belong to an homogeneous single population. And third, each individual knows the specific payoff associated with his current strategy, and learns the strategy and gets imperfect information about the payoff to another player he is randomly paired with. To explain the inter-population interaction and imitation, assume that an individual in the population of Sanchos who currently complies receives a revision opportunity. If he is paired with a defecting Sancho, which happens with probability $(1-p) / 2$, he would acknowledge that his opponent gets a higher payoff and would be willing to switch to defection. This willingness is known as the conditional imitation rate and is denoted by $r_{\mathrm{CD}}^{\mathrm{ss}}$. However, contrary to the standard two-population evolutionary game, he could also be paired with a defecting Quixote, with probability $(1-q) / 2$. He knows his current payoff, and becomes aware of his opponent's. Not knowing that they have different preferences/payoff matrices, ${ }^{7}$ the Sancho would be willing to abandon compliance if he acknowledges that the defecting Quixotes gets a higher payoff (i.e. the conditional imitation rate $r_{\mathrm{CD}}^{\mathrm{SQ}}$ is positive). Thus, the conditional switch rate from compliance to defection in the population of Sanchos has two terms:

$$
\rho_{\mathrm{CD}}^{\mathrm{s}}=\frac{1-p}{2} r_{\mathrm{CD}}^{\mathrm{ss}}+\frac{1-q}{2} r_{\mathrm{CD}}^{\mathrm{sQ}} .
$$

Considering pairwise proportional imitation, the conditional imitation rate or the willingness of an individual in population $h$ playing strategy $i$ to imitate an agent in population $k$ playing strategy $j$, can be described as proportional to the gap between the two payoffs if positive: ${ }^{8} r_{i j}^{h k}=\left[\pi_{j}^{k}-\pi_{i}^{h}\right]_{+}$where function $[d]_{+}$returns $d$ if $d>0$ or 0 otherwise. Taking this definition into account, and repeating the same reasoning, the conditional imitation rates between strategies for the two populations read:

$$
\begin{aligned}
& \rho_{\mathrm{CD}}^{\mathrm{s}}=\frac{1-p}{2} r_{\mathrm{CD}}^{\mathrm{sS}}+\frac{1-q}{2} r_{\mathrm{CD}}^{\mathrm{SQ}}=\frac{1-p}{2}(9 y+1)+\frac{1-q}{2}[9 y-1]_{+}, \\
& \rho_{\mathrm{CD}}^{\mathrm{Q}}=\frac{1-p}{2} r_{\mathrm{CD}}^{\mathrm{QS}}+\frac{1-q}{2} r_{\mathrm{CD}}^{\mathrm{QQ}}=\frac{1-p}{2}[9 y-6]_{+}+\frac{1-q}{2}[9 y-8]_{+}, \\
& \rho_{\mathrm{DC}}^{\mathrm{S}}=\frac{p}{2} r_{\mathrm{DC}}^{\mathrm{SS}}+\frac{q}{2} r_{\mathrm{DC}}^{\mathrm{SQ}}=\frac{q}{2}[6-9 y]_{+}, \\
& \rho_{\mathrm{DC}}^{\mathrm{Q}}=\frac{p}{2} r_{\mathrm{DC}}^{\mathrm{QS}}+\frac{q}{2} r_{\mathrm{DC}}^{\mathrm{QQ}}=\frac{p}{2}[1-9 y]_{+}+\frac{q}{2}[8-9 y]_{+} .
\end{aligned}
$$

It is worth noticing that the switch rate from defection to compliance in the population of Sanchos, given in (6), is not necessarily null as in the standard two-population

\footnotetext{
7 Only if a Sancho is paired with a Quixote and both play the same strategy would they realize that they have different payoff matrices. However, this match will have no influence on the dynamics as there will be no switch from one strategy to another.

8 The standard argument behind this assumption is the imperfect information concerning this gap. Thus, the larger the difference in payoffs the more likely the agents perceive it and switch strategies. We drop the $y$ argument from functions $\pi_{i}^{h}(y), \pi_{j}^{k}(y)$ and consequently from function $r_{i j}^{h k}(y)$, for simplicity.
} 
evolutionary game. A defecting Sancho never imitates a compliant Sancho, $r_{\mathrm{DC}}^{\mathrm{SS}}=0$. However, if the compliance rate in the global population is sufficiently small, $y<$ $2 / 3$, then a compliant Quixote obtains a payoff even higher than a defecting Sancho, and the latter would feel inclined to comply if paired with the former, $r_{\mathrm{DC}}^{\mathrm{se}}>0$. This opens up the possibility for an equilibrium with partial compliance among Sanchos.

From the described revision protocol and the dynamics in (2)-(3) one gets the mean dynamics for the ratios of compliant Sanchos and Quixotes:

$$
\begin{aligned}
& \dot{p}=\frac{1-p}{2}\left\{p r_{\mathrm{DC}}^{\mathrm{SS}}+q r_{\mathrm{DC}}^{\mathrm{SQ}}\right\}-\frac{p}{2}\left\{(1-p) r_{\mathrm{CD}}^{\mathrm{SS}}+(1-q) r_{\mathrm{CD}}^{\mathrm{se}}\right\}, \\
& \dot{q}=\frac{1-q}{2}\left\{q r_{\mathrm{DC}}^{\mathrm{QQ}}+p r_{\mathrm{DC}}^{\mathrm{SC}}\right\}-\frac{q}{2}\left\{(1-q) r_{\mathrm{CD}}^{\mathrm{QQ}}+(1-p) r_{\mathrm{CD}}^{\mathrm{OS}}\right\} .
\end{aligned}
$$

From (4)-(7) we can distinguish four regions for this dynamics within the interval $[0,1]$. In region I, with $y \in(8 / 9,1]$, no agent will switch from defection to compliance. Within region II, with $y \in(2 / 3,8 / 9), r_{\mathrm{DC}}^{\mathrm{QQ}}>0$ and defecting Quixotes will switch to compliance but only when paired with compliant Quixotes. Within region III $_{\alpha>1 / 2}$, with $y \in(1 / 9,2 / 3), r_{\mathrm{DC}}^{\mathrm{QQ}}>0, r_{\mathrm{DC}}^{\mathrm{SQ}}>0$, and hence compliant Quixotes will be imitated by defecting Quixotes and, more importantly, by defecting Sanchos. Finally, in region IV, with $y \in[0,1 / 9)$, compliance always pays more than defection, except when two Sanchos are paired. Taking these regions into account, the imitative dynamics (8)-(9) can be summarized in the phase diagram in Figure 1. This figure shows the two unstable equilibria in pure strategies: complete compliance among Sanchos and Quixotes, $\left(p^{*}, q^{*}\right)=(1,1)$, or defection in either population $\left(p^{*}, q^{*}\right)=(0,0)$. Moreover, we observe a mixed-strategy asymptotically stable fixed point of the dynamics in $\left(p^{*}, q^{*}\right)=(0.141048,1)$. This equilibrium is characterized by Quixotes following a pure strategy (compliance), while the population of Sanchos plays mixed strategies, complying $14 \%$ of the time. That is, an equilibrium with full compliance among Quixotes and partial compliance among Sanchos. ${ }^{9}$

According to this example, inter-population interaction and imitation creates the possibility that some Sanchos, who (in the standard two-population setting) would never imitate compliant Sanchos, might now imitate compliant Quixotes. A mixedstrategy equilibrium of the form $q^{*}=1$ and $p^{*} \in(0,1)$ arises. However, the next example highlights that inter-population interaction and imitation can lead to a mixedstrategy equilibrium of a different type: $q^{*} \in(0,1)$ and $p^{*}=0$. Assuming the payoff matrix for Sanchos in S-I, consider a new type of Quixotes whose payoff matrix reads:

\footnotetext{
9 To compute this mixed-strategy equilibrium, notice that in region $\mathbf{I I I}_{\alpha>1 / 2}, r_{\mathrm{CD}}^{\mathrm{QQ}}=r_{\mathrm{CD}}^{\mathrm{QS}}=0$ and hence, $\dot{q}=(1-q)\left\{q r_{\mathrm{DC}}^{\mathrm{QQ}}+p r_{\mathrm{DC}}^{\mathrm{QS}}\right\} / 2$ which is positive unless $q$ reaches 1 . When all Quixotes comply, the dynamic equation for $p$, in (8), vanishes for a unique value satisfying that $y$ remains in this region: $p^{*}=0.141048$.
} 


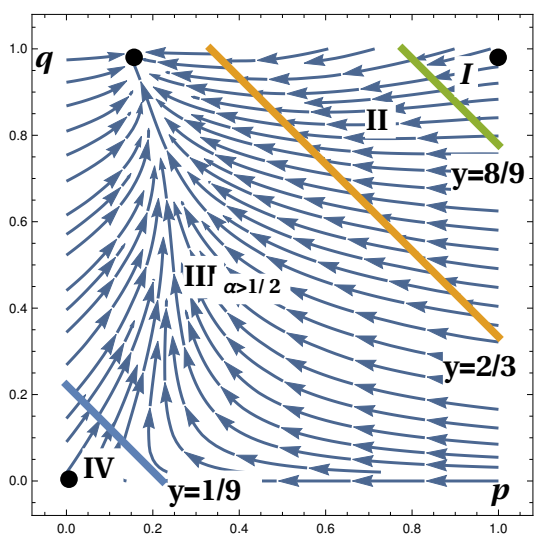

Fig. 1 Phase diagram in example S-I/Q-I.

\begin{tabular}{c|c|c} 
& $\mathrm{C}$ & $\mathrm{D}$ \\
\hline $\mathrm{C}$ & $(14,14)$ & $(4,15)$ \\
$\mathrm{D}$ & $(15,4)$ & $(-4,-4)$ \\
\hline
\end{tabular}

Again, Sanchos are characterized by a prisoner's dilemma game and Quixotes by a chicken game. This new type of Quixotes obtain 4 more units from compliance (instead of 7) and 5 less units from defection (instead of 2). The new chicken game is characterized by the same Nash equilibrium in mixed strategies, $\Delta=8 / 9$. Following the same reasoning as in the previous example, the imitative dynamics is described in the phase diagram in Figure 2. Together with the two unstable equilibria $(1,1)$ and $(0,0)$, a mixed-strategy equilibrium is obtained at $\left(p^{*}, q^{*}\right)=(0,0.828956)$. In this equilibrium, Sanchos follow a pure defecting strategy and Quixotes follow a mixed strategy, complying $82 \%$ of the time. This equilibrium is characterized by partial compliance among Quixotes and defection among Sanchos.

Note that although the compliance rate in the population of Sanchos is zero at the equilibrium, it is not necessarily so along the whole trajectory towards the equilibrium. As shown in Figure 2, starting from an initial situation of very low compliance in both populations, compliant Quixotes are paid more than defecting Sanchos. Therefore, defecting Sanchos switch to compliance when paired with compliant Quixotes. Nevertheless, this imitation process is of limited duration. As the global compliance rate increases, the excess reward of compliant Quixotes progressively disappears, leading the population of Sanchos towards defection.

The two examples lead to a unique mixed-strategy asymptotically stable fixed point. In example S-I/Q-I, the population of Quixotes follows the compliance strategy while Sanchos are characterized by a mixed strategy. Conversely, in example S-I/QII, the population of Sanchos follows the defecting strategy while the behaviour of 


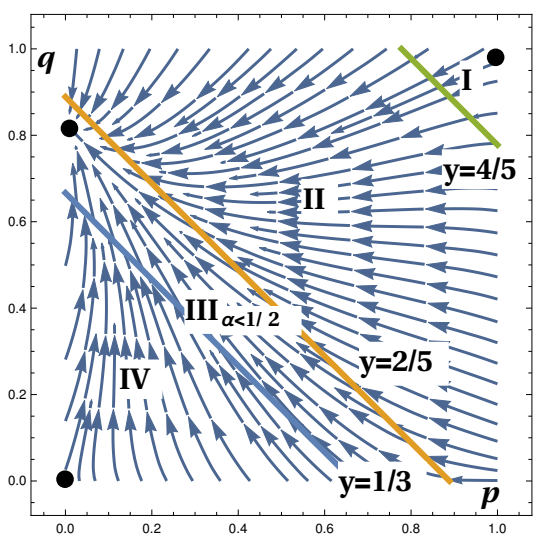

Fig. 2 Phase diagram in example S-I/Q-II

Quixotes is characterized by a mixed strategy. Next we analyze the conditions that lead to one type of solution or the other in a general setting.

\section{A general model}

The payoff matrix for the population of Sanchos can be expressed as:

\begin{tabular}{c|c|c} 
& $\mathrm{C}$ & $\mathrm{D}$ \\
\hline $\mathrm{C}$ & $(1,1)$ & $(1-d, 1+c-d)$ \\
$\mathrm{D}$ & $(1+c-d, 1-d)$ & $(1-\phi, 1-\phi)$ \\
\hline
\end{tabular}

This game has a prisoner's dilemma structure under condition:

$$
0<\phi<d<c
$$

Furthermore, we also assume that defection is more strongly preferred if the opponent complies: $d-\phi>c-d$, or equivalently, $\sigma=\phi+c-2 d>0$.

Quixotes obtain an excess reward from compliance, given by $A \geq 0$, and a lower payoff associated with defection, $B \geq 0$. Hence, their payoff matrix is:

\begin{tabular}{c|c|c} 
& $\mathrm{C}$ & $\mathrm{D}$ \\
\hline $\mathrm{C}$ & $(1+A, 1+A)$ & $(1-d+A, 1+c-d-B)$ \\
$\mathrm{D}$ & $(1+c-d-B, 1-d+A)$ & $(1-\phi-B, 1-\phi-B)$ \\
\hline
\end{tabular}

We can interpret the term $\varepsilon=A+B>0$, as a "quantitative" measure of the gap between Sanchos and Quixotes. This measure adds together how much better compliance is and how much worse defection is for Quixotes. Then $A$ and $B$ can be rewritten as $A=\alpha \varepsilon$ and $B=(1-\alpha) \varepsilon$ with $\alpha \in[0,1]$. The new parameter $\alpha$ is a "qualitative" measure which discriminates between a great joy from compliance $(\alpha \rightarrow 1)$ or 
a strong discomfort associated with transgression $(\alpha \rightarrow 0)$. Thus, $\varepsilon$ represents how different the two population are, while $\alpha$ describes in which way they differ.

Under this specification, the snowdrift structure arises if defection is preferred when the opponent complies, but not when the opponent also defects:

$$
0<d-\phi<\varepsilon<c-d \text {. }
$$

Note that the example S-I/Q-I corresponds to the values $d=1, c=2, \phi=9 / 10, \varepsilon=$ $9 / 10, \alpha=7 / 9$, multiplying all payoffs by 10 . And the example $\mathrm{S}-\mathrm{I} / \mathrm{Q}-\mathrm{II}$ corresponds to the same parameters values except for $\alpha=4 / 9$.

Following the same reasoning as in the two previous examples, when individuals in one population play a game against individuals in their own as well as in the other population, (i.e. under inter-population interaction), the $4 \times 4$ matrix:

$$
\left(\begin{array}{cccc}
1 & 1-d & 1 & 1-d \\
1+c-d & 1-\phi & 1+c-d & 1-\phi \\
1+\alpha \varepsilon & 1-d+\alpha \varepsilon & 1+\alpha \varepsilon & 1-d+\alpha \varepsilon \\
1+c-d-(1-\alpha) \varepsilon & 1-\phi-(1-\alpha) \varepsilon & 1+c-d-(1-\alpha) \varepsilon & 1-\phi-(1-\alpha) \varepsilon
\end{array}\right)
$$

determines the vector of payoffs:

$$
\pi(y)=\left(\begin{array}{c}
\pi_{\mathrm{C}}^{\mathrm{s}}(y) \\
\pi_{\mathrm{D}}^{\mathrm{s}}(y) \\
\pi_{\mathrm{C}}^{\mathrm{Q}}(y) \\
\pi_{\mathrm{D}}^{\mathrm{Q}}(y)
\end{array}\right)=\left(\begin{array}{c}
1-d+d y \\
1-\phi+y(\phi+c-d) \\
1-d+d y+\alpha \varepsilon \\
1-\phi+y(\phi+c-d)-(1-\alpha) \varepsilon
\end{array}\right)
$$

And next proposition computes the unique Nash equilibrium for this game.

Proposition 1 The Nash equilibrium of the two-population game involving Sanchos and Quixotes, who share the same strategy set $\{C, D\}$, and with payoffs as described in (12), is: ${ }^{10}$

$$
\left(p^{N E}, q^{N E}\right)=\left\{\begin{array}{l}
(0,1) \quad \text { if } \varepsilon \geq \frac{c-\phi}{2}, \\
(0,2 \Delta) \text { if } \varepsilon<\frac{c-\phi}{2},
\end{array} \quad \Delta=\frac{\varepsilon-(d-\phi)}{\sigma} .\right.
$$

Proof The payoffs in (12) imply $\pi_{\mathrm{D}}^{\mathrm{s}}(y)>\pi_{\mathrm{C}}^{\mathrm{s}}(y)$, for all $y \in[0,1]$, and hence compliance is a strictly dominated strategy for Sanchos. Thus at any state $\mathbf{x}=$ $(p, 1-p, q, 1-q)$, the best reaction function for Sanchos reads: $B^{\mathrm{s}}(\mathbf{x})=(0,1)$.

For Quixotes, computing the value of $q$ at which $\pi_{\mathrm{D}}^{\mathrm{Q}}(y)=\pi_{\mathrm{C}}^{\mathrm{S}}(y)$ for the expressions in (12), the best reaction function can be written as: $B^{\mathrm{Q}}(\mathbf{x})=(2(2 \Delta-p), 1-$ $2(2 \Delta-p))$. From these two reaction functions, and the fact that $q^{\mathrm{NE}}$ cannot be greater than one, the Nash equilibrium in (13) follows.

\footnotetext{
${ }^{10}$ For the parameters' values above, the Nash equilibrium would be $\left(p^{\mathrm{NE}}, q^{\mathrm{NE}}\right)=(0,1)$.
} 
Under pairwise imitation, the imitative dynamics is governed by the differential equations in (2) and (3), together with the swift rates between strategies defined by:

$$
\begin{aligned}
& \rho_{\mathrm{CD}}^{\mathrm{s}}=\frac{1-p}{2}\left[y+\frac{d-\phi}{\sigma}\right]_{+}+\frac{1-q}{2}\left[y-\Delta_{2}\right]_{+}, \\
& \rho_{\mathrm{CD}}^{\mathrm{Q}}=\frac{1-p}{2}\left[y-\Delta_{1}\right]_{+}+\frac{1-q}{2}[y-\Delta]_{+}, \\
& \rho_{\mathrm{DC}}^{\mathrm{s}}=\frac{q}{2}\left[\Delta_{1}-y\right]_{+}, \\
& \rho_{\mathrm{DC}}^{\mathrm{Q}}=\frac{p}{2}\left[\Delta_{2}-y\right]_{+}+\frac{q}{2}[\Delta-y]_{+} .
\end{aligned}
$$

with

$$
\Delta_{1}=\Delta-(1-\alpha) \frac{\varepsilon}{\sigma}, \quad \Delta_{2}=\Delta-\alpha \frac{\varepsilon}{\sigma} .
$$

Note that $\Delta \geq \max \left\{\Delta_{1}, \Delta_{2}\right\}$ and $\Delta_{1} \gtrless \Delta_{2}$ when $\alpha \gtrless 1 / 2$.

The dynamics depends on the value of $y$ and on how it compares to $\Delta, \Delta_{1}$ and $\Delta_{2}$. Figure 3 defines five regions depending on the conditional imitation rates of defecting $h$ individuals paired with compliant $k$ individuals, $r_{\mathrm{DC}}^{h k}, h, k \in\{S, Q\} .{ }^{11}$

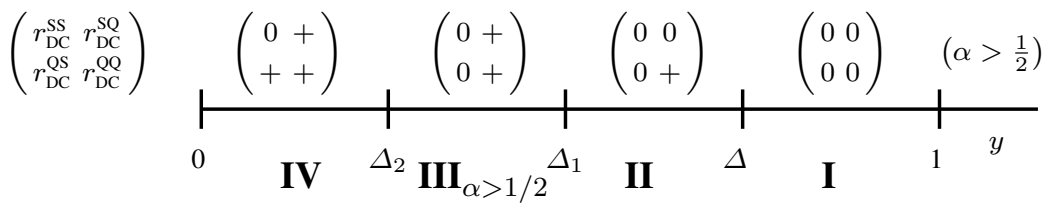

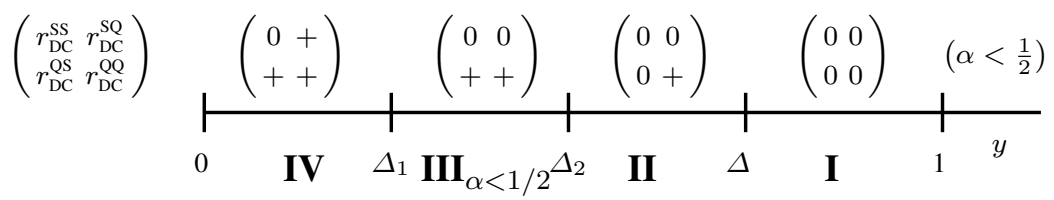

Fig. 3 Conditional imitation rates from defection to compliance when $\alpha>1 / 2$ (up) or $\alpha<1 / 2$ (down)

In region $\mathbf{I}, \dot{p}<0$ if $p \in(0,1)$ and $\dot{q}<0$ if $q \in(0,1)$, hence, an equilibrium with some compliance is not feasible in this region. The unstable equilibria $\left(p^{*}, q^{*}\right)=(1,1)$ and $\left(p^{*}, q^{*}\right)=(0,0)$ lie in regions $\mathbf{I}$ and $\mathbf{I V}$, respectively. A

\footnotetext{
${ }^{11}$ In the interior of these intervals $r_{\mathrm{DC}}^{h k}>0 \Rightarrow r_{\mathrm{CD}}^{k h}=0$, and similarly $r_{\mathrm{DC}}^{h k}=0 \Rightarrow r_{\mathrm{CD}}^{k h}>0$, for $h, k \in\{S, Q\}$. Consequently, the conditional imitation rates for compliant $h$ individuals paired with defecting $k$ individuals can be immediately obtained by switching 0 by + (and vice versa), and transposing the matrices in Figure 3.
} 
mixed-strategy equilibrium with full compliance among Quixotes and partial compliance among Sanchos (like in example S-I/Q-I) requires that some defecting Sanchos switch to compliance when paired with compliant Quixotes, i.e. it requires $r_{\mathrm{DC}}^{\mathrm{SQ}}>0$, or equivalently, $y<\Delta_{1}$. This corresponds to regions IV and III $_{\alpha>1 / 2}$ in Figure 3. Conversely, an equilibrium with partial compliance among Quixotes and pure defection among Sanchos, requires the opposite condition: $y>\Delta_{1}$. This corresponds to regions II and III $_{\alpha<1 / 2}$ in Figure 3. These two types of equilibrium are characterized in the following proposition.

Proposition 2 The inter-population interaction and imitation makes possible a mixedstrategy equilibrium of two types. Under condition

$$
\varepsilon \alpha>\frac{c-\phi}{2}
$$

the equilibrium shows full compliance within the population of Quixotes, $q_{S Q}^{*}=1$, and partial compliance within the population of Sanchos: ${ }^{12}$

$$
p_{s Q}^{*}=\frac{-(c-d)+\sqrt{(d-\phi)^{2}+2 \alpha \varepsilon \sigma}}{\sigma} \in(0,1) .
$$

Under the opposite condition

$$
\varepsilon \alpha<\frac{c-\phi}{2}
$$

the equilibrium is characterized by defection within the population of Sanchos, $p_{Q}^{*}=$ 0 , and partial compliance within the population of Quixotes:

$$
q_{Q}^{*}=1+\Delta-\sqrt{(1-\Delta)^{2}+2(1-\alpha) \varepsilon / \sigma}<1 .
$$

The equality $\varepsilon \alpha=(c-\phi) / 2$ would lead to a $\left(p^{*}, q^{*}\right)=(0,1)$ equilibrium with both populations playing pure strategies: Quixotes compliance and Sanchos defection.

Proof See Appendix A.

Remark 1 To guarantee $q_{\mathrm{Q}}^{*}>0$ in (21), condition (11) is strengthen to:

$$
\frac{2}{1+\alpha}(d-\phi)<\varepsilon<c-d .
$$

The results collected in the two previous examples, and in Proposition 2 and Remark 1 are gathered together in Figure 4, which depicts the equilibria in the $\varepsilon-\alpha$ parameters' space. The existence of an equilibrium with positive compliance requires a sufficiently large absolute distance between Quixotes and Sanchos, given by the left inequality in (22). In Figure 4, the shadow region down-left of the dashed line represents pairs $(\varepsilon, \alpha)$ for which this condition is not fulfilled and no equilibrium is feasible.

\footnotetext{
12 Subscript $S Q$ indicates that individuals from both populations comply. Subscript $Q$ is used to highlight that only Quixotes comply.
} 


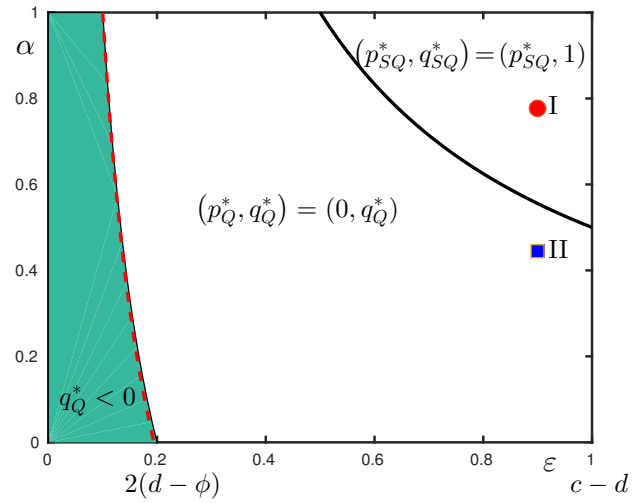

Fig. 4 Distinct equilibria depending on $\varepsilon$ and $\alpha,(c=2, d=1, \phi=9 / 10)$.

According to condition (18), some Sanchos can find it attractive to comply in the case of inter-population interaction and imitation. This type of equilibrium, with compliant Quixotes plus partially compliant Sanchos, is reached above and to the right of the solid line in Figure 4. Example S-I/Q-I lies in this region (highlighted by the dot I). The absolute distance between Sanchos and Quixotes, $\varepsilon$, should not necessarily be too large if this difference focuses on greater payoffs for compliance, i.e. if $\alpha$ approaches 1 . But this absolute distance needs to be large when it mainly implies stronger penalties from defection, ${ }^{13} \alpha$ close to 0 .

By contrast, condition (18) is reversed to (20), and the second type of equilibrium emerges, below and to the left of of the solid line in Figure 4. In this equilibrium only some Quixotes comply. It occurs when the absolute gap between Sanchos and Quixotes is not excessively wide ( $\varepsilon$ small); and/or if this difference is biased towards a stronger aversion to defection ( $\alpha$ small). Example S-I/Q-II lies in this region (highlighted by the square II).

Remark 2 In the two types of equilibrium presented in Proposition 2, one population plays a mixed strategy while the other population plays a pure strategy. Under our formulation, a mixed-mixed equilibrium with $p^{*} \in(0,1)$ and $q^{*} \in(0,1)$, is not feasible. If some Sanchos comply, it must be the case that all Quixotes do also comply. And conversely, if some Quixotes defect, then no Sancho complies.

Proof From expression (19), condition $p_{\mathrm{SQ}}^{*}>0$ is equivalent to $\alpha \varepsilon>(c-\phi) / 2$, or condition (18). Similarly, from the definition in (21), the condition $q_{\mathrm{Q}}^{*}<1$ is equivalent to $\alpha \varepsilon<(c-\phi) / 2$, or the reverse of condition (20). Obviously the two requirements cannot be fulfilled at the same time.

${ }^{13}$ In fact, for the parameters' values considered, it is not feasible for $\alpha<1 / 2$. 
To clarify this remark, assume an initial situation of $(p, q)=(0,1)$. If condition (18) is satisfied, compliant Quixotes do better than defecting Sanchos or Quixotes. ${ }^{14}$ Therefore they never switch to defection: $r_{\mathrm{CD}}^{\mathrm{QQ}}=r_{\mathrm{CD}}^{\mathrm{QS}}=0$ and $q$ remains equal to $q_{\mathrm{SQ}}^{*}=1$. Moreover, since defecting Sanchos do worse than compliant Quixotes, some of them will switch and imitate the compliant behavior of Quixotes, until the share $p_{\mathrm{SQ}}^{*}>0$.

Conversely, let us assume that at the same initial point $(p, q)=(0,1)$, the opposite condition (20) is satisfied. Then, defecting Sanchos do not imitate compliant Quixotes (and they never imitate compliant Sanchos). The defecting Sanchos never switch to compliance and $p$ remains equal to $p_{\mathrm{Q}}^{*}=0$. By contrast, compliant Quixotes would switch and stop compliance when paired with defecting Sanchos. This explains a compliance rate for Quixotes $q_{\mathrm{Q}}^{*}<1$. As the share of compliant Quixotes decays, so does their disadvantage with respect to defecting Sanchos. However, under condition (20), defecting Sanchos still do better than compliant Quixotes even at the equilibrium, $\pi_{\mathrm{C}}^{\mathrm{Q}}\left(q_{\mathrm{Q}}^{*} / 2\right)<\pi_{\mathrm{D}}^{\mathrm{S}}\left(q_{\mathrm{Q}}^{*} / 2\right)$. Consequently, Sanchos still have no incentive to abandon their dominant defecting strategy.

Proposition 2 and Remark 2 establish the existence of an equilibrium with one population playing a mix strategy (and the other a pure strategy). Next proposition states that this equilibrium is the unique stable equilibrium.

Proposition 3 The equilibrium $\left(p_{S Q}^{*}, 1\right)$ under condition (18), or $\left(0, q_{Q}^{*}\right)$ under the opposite condition (20), is the unique asymptotically stable fixed point of the evolutionary dynamics.

Proof The stability of the equilibria can be tackled based on two approaches. A first approach is the direct method of Lyapunov. Let's denote by $V\left(p, q, p^{*}, q^{*}, t\right)$ the euclidean distance from $(p, q)$ to the equilibrium $\left(p^{*}, q^{*}\right)$ at time $t$, which is locally positive definite (lpd) and decrescent. Then the equilibrium $\left(p^{*}, q^{*}\right)$ would be uniformly locally asymptotically stable if $-\dot{V}\left(p, q, p^{*}, q^{*}, t\right)$ is lpd.

We have computed functions $-\dot{V}\left(p, q, p_{\mathrm{SQ}}^{*}, q_{\mathrm{SQ}}^{*}, t\right)$ for the example S-I/Q-I and $-\dot{V}\left(p, q, p_{\mathrm{Q}}^{*}, q_{\mathrm{Q}}^{*}, t\right)$ for the example $\mathrm{S}-\mathrm{I} / \mathrm{Q}-\mathrm{II}$. Although we cannot proof analytically that these functions are lpd, it can be numerically inferred by plotting both functions. Figure 5 shows the level curves for functions $\dot{V}\left(p, q, p_{\mathrm{SQ}}^{*}, q_{\mathrm{sQ}}^{*}, t\right)$ and $\dot{V}\left(p, q, p_{\mathrm{Q}}^{*}, q_{\mathrm{Q}}^{*}, t\right)$ which only take negative values.

Moreover, it is easy to prove that condition

$$
-\dot{V} \equiv-\left(\frac{\partial V}{\partial p}, \frac{\partial V}{\partial q}\right) \cdot(\dot{p}, \dot{q})^{\prime}>0
$$

${ }^{14} \pi_{\mathrm{C}}^{\mathrm{Q}}(1 / 2)-\pi_{\mathrm{D}}^{\mathrm{S}}(1 / 2)=\alpha \varepsilon-(c-\phi) / 2$. Then, under condition (18) it holds true that $\pi_{\mathrm{C}}^{\mathrm{Q}}(1 / 2)>$ $\pi_{\mathrm{D}}^{\mathrm{S}}(1 / 2)$. Moreover, it is always true that $\pi_{\mathrm{D}}^{\mathrm{S}}(y)>\pi_{\mathrm{C}}^{\mathrm{S}}(y)$. 

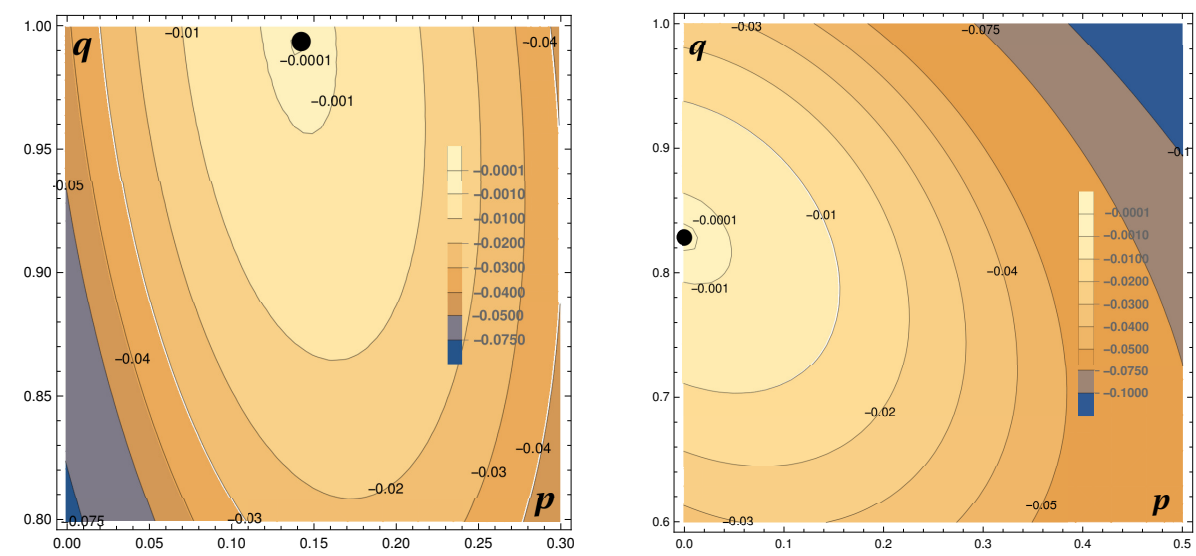

Fig. $5 \dot{V}\left(p, q, p^{\mathrm{SQ}}, q^{\mathrm{SQ}}\right)$ and $\dot{V}\left(p, q, p^{\mathrm{Q}}, q^{\mathrm{Q}}\right)$

is equivalent to say that the direction of the dynamics makes an acute angle with the direction pointing from the state $(p, q)$ to the equilibrium $\left(p^{*}, q^{*}\right)$. This is also clear in the vector fields shown in Figures 1 and 2.

Local asymptotic stability of the two type of equilibrium can be analytically proved considering the alternative approach of the Lyapunov indirect method. If the linearized system around the equilibrium $\left(p^{*}, q^{*}\right)$ is a uniformly asymptotically stable equilibrium point, then $\left(p^{*}, q^{*}\right)$ is a locally asymptotically stable equilibrium point of the dynamic system. For each equilibrium type, in Appendix B we prove that the two eigenvalues of the Jacobian matrix of the system of differential equations (within the region where the equilibrium is located) are in the left half complex plane (have negative real part).

Propositions 2 and 3 establish the existence of a unique stable equilibrium, given by $\left(p_{\mathrm{sQ}}^{*}, 1\right)$ under condition $(18)$, or $\left(0, q_{\mathrm{Q}}^{*}\right)$ under the opposite condition (20). Next proposition compares these two type of equilibrium to the unstable Nash equilibrium in (13). This would be the asymptotically stable equilibrium in the case of interpopulation interaction but no inter-population imitation. That is, if individuals from one population would only imitate individuals from their same population.

Proposition 4 Under condition (18), the asymptotically stable equilibrium, characterized by compliant Quixotes and partially compliant Sanchos, satisfies: $p_{S Q}^{*}>p^{N E}=$ 0 and $q_{S Q}^{*}=q^{N E}=1$.

Under the opposite condition (20), the stable equilibrium, characterized by partially compliant Quixotes and defecting Sanchos, satisfies: $p_{Q}^{*}=p^{N E}=0$ and $q_{Q}^{*}<q^{N E}$.

Proof See Appendix C. 
Under condition (18), the asymptotically stable equilibrium is characterized by a positive share of Sanchos who imitate the compliant behavior of Quixotes, when their payoff would be higher following the defecting strategy in their Nash equilibrium. In this situation every individual in the population of Quixotes ends up complying, as it is also marked by their Nash equilibrium.

By contrast, under the alternative condition (20), when only Quixotes comply, they comply less than what they would have complied had the Nash equilibrium be reached (i.e. without inter-population imitation). The reason is that there exists a positive share of Quixotes which imitate the defecting behavior of Sanchos, when it would be in their best interest not to do so. Under this condition, the population of Sanchos follows its Nash equilibrium or dominant defecting strategy.

Remark 3 We can define the average payoffs for Sanchos and for Quixotes as:

$$
\bar{\pi}^{\mathrm{s}}(p, q)=\pi_{\mathrm{C}}^{\mathrm{s}}(y) p+\pi_{\mathrm{D}}^{\mathrm{s}}(y)(1-p), \quad \bar{\pi}^{\mathrm{Q}}(p, q)=\pi_{\mathrm{C}}^{\mathrm{Q}}(y) q+\pi_{\mathrm{D}}^{\mathrm{Q}}(y)(1-q) .
$$

Then, the asymptotically stable equilibrium under condition (18) provides a higher average payoff to Quixotes, because Sanchos comply above the Nash equilibrium. Likewise, under condition (20) Sanchos attain a smaller average payoff, because Quixotes comply below the Nash equilibrium.

Proof See Appendix D.

\section{Conclusions}

The paper analyzes a social dilemma involving two types of individuals who share the same strategy set and differ in their payoffs. For Sanchos, the game has a prisoner's dilemma structure, and for Quixotes the dilemma is described as a snowdrift game. If a one-population evolutionary game were assumed considering only Sanchos, the evolutionary dynamics would converge to the defecting Nash equilibrium characteristic from a prisoner's dilemma. By contrast, if the population was exclusively composed of Quixotes, the evolutionary dynamics would converge towards an equilibrium with positive shares of compliant and defecting Quixotes, defined by the Nash equilibrium in mixed strategies of the snowdrift game.

Our approach analyzes the social dilemma as a two-population evolutionary game, introducing a setting in between a symmetric evolutionary game and an asymmetric evolutionary game. The game considers inter-population interaction and it randomly pits players from a given population against themselves, as well as against agents from the other population. 
Evolutionary dynamics is defined considering an imitative revision protocol, specifically, pairwise proportional imitation. In the standard two-population evolutionary game, agents are only paired with individuals within their own population. If an individual revising his strategy is paired with an individual of the same population playing a different strategy, he will switch to this strategy with a conditional imitation rate, as long as it provides a higher payoff. Our approach allows for the possibility of inter-population imitation. Each individual knows the payoff associated with his current strategy, learns the strategy of another player he is randomly paired with, and gets imperfect information about this agent's payoff. The wider the gap between the payoffs, the clearer it is perceived, and the more likely a change in strategy. However, agents are unaware of the heterogeneity in payoffs, and ignore the existence of two distinct populations. Thus, a revising agent imitates an alternative strategy when played by either an individual within his own population or the other population.

Our main result is that the proposed setting makes possible stable mixed-strategy equilibria, that need not be and indeed are not pure. Specifically, the imitative dynamics admits two mixed-strategy asymptotically stable rest points: an equilibrium with compliant Quixotes and partially compliant Sanchos, or an equilibrium with defecting Sanchos and partially compliant Quixotes. In both types of equilibria, individuals in one population play a pure strategy, while individuals in the other population are characterized by a mixed strategy. An equilibrium with full compliance among Quixotes and partial compliance among Sanchos takes place if the excess reward that Quixotes attain from compliance is large. That is, if Quixotes strongly differ from Sanchos, and/or this difference focuses on greater payoffs from compliance rather than greater penalties from defection. The equilibrium results in full defection among Sanchos and partial compliance among Quixotes in the opposite case, when the two populations are similar, or they differ mainly from a strong distaste for defection.

Furthermore, we also prove that the asymptotically stable equilibria are not Nash equilibria. We observe that in the stable equilibrium in which only Quixotes comply, they comply below the Nash equilibrium. Conversely, when also Sanchos comply, they exceed the zero compliance under the Nash equilibrium.

We have presented the inter-population interaction and imitation as an extreme setting opposed to the standard evolutionary game. In the latter, players have full information on their own and their opponents payoff matrices. Conversely, we have assumed that a player only knows his current payoff and the payoff of the player he is paired with. An interesting extension might consider intermediate situations in which individuals do not fully recognize, but neither do they completely fail to acknowledge, the other individuals' type. Consequently, a player in one population would also imitate individuals from the other population, but to a lesser extent. A second in- 
teresting extension would be to consider that the population is not necessarily divided in two equal halves between Sanchos and Quixotes.

Finally, we have considered a particular structure for the payoff matrix of Quixotes with respect to Sanchos (higher reward to compliance and lower reward to defection). Alternative specifications could give rise to different results. In particular, it would be worthy to explore the possibility of a mixed-mixed equilibrium if Quixotes obtain a higher reward from compliance and also from defection.

\section{A Proof of Proposition 2}

- Equilibrium under condition (18):

Since $r_{\mathrm{DC}}^{\mathrm{SS}}=0$, the dynamics of the compliance share among Sanchos for $q=1$ reads:

$$
\dot{p}=(1-p) r_{\mathrm{DC}}^{\mathrm{SQ}}-p(1-p) r_{\mathrm{CD}}^{\mathrm{SS}}=(1-p) \sigma\left[\Delta_{1}-y-p\left(y+\frac{d-\phi}{\sigma}\right)\right] .
$$

Hence, taking into account that $y=(1+p) / 2$, then $\dot{p} /((1-p) \sigma)$ is given by the second order polynomial:

$$
-p^{2}-2 p \frac{c-d}{\sigma}+2 \frac{\alpha \varepsilon-(d-\phi)}{\sigma}-1
$$

This polynomial has one stable and one unstable real root. The stable solution defines $p_{\mathrm{SQ}}^{*}$ in (19). Since an equilibrium in this regions requires that all Quixotes comply, the share of compliance in the global population can be obtained as: $y_{\mathrm{SQ}}^{*}=\left(1+p_{\mathrm{SQ}}^{*}\right) / 2$. That is:

$$
y_{\mathrm{SQ}}^{*}=\frac{-(d-\phi)+\sqrt{(d-\phi)^{2}+2 \alpha \varepsilon \sigma}}{2 \sigma},
$$

which is always positive and lower than 1 . Furthermore, it lies within region IV or III $_{\alpha>1 / 2}$ if and only if $y_{\mathrm{SQ}}^{*} \leq \Delta_{1}$, which is satisfied under condition (18).

It is easy to show that condition (18), which guarantees the existence of this type of equilibrium, is also a necessary and sufficient condition for the share of compliant Sanchos in (19) to be positive. Moreover, $p_{\mathrm{SQ}}^{*}$ is strictly lower than 1 as long as Quixotes play a snowdrift game, which occurs under condition (11)

- Equilibrium under condition (20):

Whenever $y<\Delta$, compliant Quixotes do not abandon compliance when paired with defecting Quixotes, $r_{\mathrm{CD}}^{\mathrm{QQ}}=0$. Furthermore, since $p=0$, the dynamics for the compliance share among Quixotes reads:

$$
\dot{q}=(1-q) q r_{\mathrm{DC}}^{\mathrm{QQ}}-q r_{\mathrm{CD}}^{\mathrm{QS}}=q \sigma\left[(1-q)[\Delta-y]-\left(y-\Delta_{1}\right)\right] .
$$

Taking into account that now $y=q / 2$, then $\dot{q} /(q \sigma)$ is given by the second order polynomial:

$$
q^{2}-2 q[1+\Delta]+4 \Delta-2(1-\alpha) \frac{\varepsilon}{\sigma} .
$$

The stable root for this polynomial is given in (21).

For this solution to be feasible, it must remain within regions III $_{\alpha<1 / 2}$ or II, i.e. $y_{\mathrm{Q}}^{*}>\Delta_{1}$. After some algebra, it is easy to conclude that this inequality is equivalent to condition (20). It is also easy to prove that this is also equivalent to $q_{\mathrm{Q}}^{*}<1$. 


\section{B Proof of Proposition 3}

First, we compute the Jacobian matrix, $J(p, q)$, of the system of differential equations within the region where the equilibrium is located. Then we compute the two eigenvalues of $J\left(p^{*}, q^{*}\right)$.

The equilibrium $\left(p_{\mathrm{SQ}}^{*}, 1\right)$ which lies within region ${ }^{15} \mathbf{I I I}_{\alpha>1 / 2}$, has associated the two eigenvalues:

$$
\begin{aligned}
& \lambda_{\mathrm{SQ}}^{1}=(d-\phi)-2 \varepsilon+\sqrt{(d-\phi)^{2}+2 \alpha \varepsilon \sigma}, \\
& \lambda_{\mathrm{SQ}}^{2}=(d-\phi)^{2}+2 \alpha \varepsilon \sigma-(\sigma+c-d) \sqrt{(d-\phi)^{2}+2 \alpha \varepsilon \sigma} .
\end{aligned}
$$

After some calculus, it is easy to see that $\lambda_{\mathrm{SQ}}^{1}<0$ is equivalent to:

$$
\varepsilon>\alpha \frac{c-\phi}{2}+(1-\alpha)(d-\phi) .
$$

But since $\alpha \in[0,1]$, then from condition (18) it is immediate to conclude that $\varepsilon>\alpha \varepsilon>(c-\phi) / 2>$ $\alpha(c-\phi) / 2$. Hence condition above follows, and $\lambda_{\mathrm{SQ}}^{1}<0$.

Likewise, $\lambda_{\mathrm{SQ}}^{2}<0$ is equivalent to:

$$
\alpha \varepsilon \sigma[2 \alpha \varepsilon-4(c-d)]<(d-\phi)^{2}[(c-d)-\alpha \varepsilon] .
$$

This condition can be proved based on the fact that $p_{\mathrm{SQ}}^{*} \leq 1$ which, after some calculus, is equivalent to $2 \alpha \varepsilon-4(c-d) \leq 0$. Moreover, since $\alpha \varepsilon \leq \varepsilon<c-d$, then it is immediately obvious that the LHS of previous inequality is non-positive, while the RHS is positive, which concludes the proof of $\lambda_{\mathrm{SQ}}^{2}<0$.

The equilibrium $\left(0, q_{\mathrm{Q}}^{*}\right)$ which lies within region ${ }^{16} \mathbf{I I I}_{\alpha<1 / 2}$, has associated the two eigenvalues:

$$
\begin{aligned}
& \lambda_{\mathrm{Q}}^{1}=\frac{1}{4}\{-(c-d)-\varepsilon+\sqrt{D i s}\}, \quad \text { Dis }=(c-d)^{2}-2 \alpha \varepsilon \sigma-2(d-\phi) \varepsilon+\varepsilon^{2}, \\
& \lambda_{\mathrm{Q}}^{2}=\frac{1}{2 \sigma}\{D i s-[\sigma+\varepsilon-(d-\phi)] \sqrt{D i s}\} .
\end{aligned}
$$

It is easy to see that $\lambda_{\mathrm{Q}}^{1}<0$ is equivalent to:

$$
-2 \alpha \varepsilon \sigma-2(d-\phi) \varepsilon<2 \varepsilon(c-d) .
$$

The LHS in this inequality is negative and the RHS positive. Therefore, it always holds

Finally, $\lambda_{\mathrm{Q}}^{2}<0$ can be equivalently written as $\alpha \varepsilon-2(d-\phi)>-(c-d)$. But this condition is true as long as $q_{\mathrm{Q}}^{*}$ in (21) takes a positive value.

\section{Proof of Proposition 4}

Under condition (20) the stable equilibrium is $\left(0, q_{\mathrm{Q}}^{*}\right)$, and the Nash equilibrium is $\left(0, q^{\mathrm{NE}}\right)$. Obviously $p^{\mathrm{NE}}=p_{\mathrm{Q}}^{*}=0$. Moreover, taking into account (21) and (13), one gets that $q^{\mathrm{NE}}>q_{\mathrm{Q}}^{*}$ if and only if:

$$
\sqrt{(1-\Delta)^{2}+2(1-\alpha) \varepsilon / \sigma}>(1+\Delta)-2 \Delta=1-\Delta,
$$

which immediately holds.

Conversely, under condition (18) the stable equilibrium is $\left(p_{\mathrm{SQ}}^{*}, 1\right)$, and the Nash equilibrium is $\left(0, q^{\mathrm{NE}}\right)$. From (18) immediately follows that $p_{\mathrm{SQ}}^{*}>p^{\mathrm{NE}}=0$. However, from condition (18) and the fact that $\alpha \in[0,1]$ one gets:

$$
\varepsilon \geq \alpha \varepsilon>\frac{c-\phi}{2} .
$$

This inequalities imply $2 \Delta>1$ and, taking into account (13), $q^{\mathrm{NE}}=1$.

15 A similar analysis could be done if this equilibrium had lied in region IV.

16 A similar analysis could be done if this equilibrium had lied in region II. 


\section{Proof of Remark 3}

It is easy to see that:

$$
q^{\mathrm{NE}}<1 \text { if } \varepsilon<\frac{c-\phi}{2}, \text { and } q^{\mathrm{NE}}=1 \text { if } \varepsilon \in\left(\frac{c-\phi}{2}, \frac{c-\phi}{2 \alpha}\right) .
$$

Thus, under condition (18), $\varepsilon>\alpha \varepsilon>(c-\phi) / 2$ and $q^{\mathrm{NE}}=1$. The gap in the average payoff for Quixotes between the asymptotically stable equilibrium and the Nash equilibrium reads:

$$
\bar{\pi}^{\mathrm{Q}}\left(p_{\mathrm{SQ}}^{*}, 1\right)-\bar{\pi}^{\mathrm{Q}}(0,1)=\frac{d p_{\mathrm{SQ}}^{*}}{2}>0 .
$$

Under the alternative condition (20), although $p^{\mathrm{NE}}=0, q^{\mathrm{NE}}$ can be equal or lower than 1 .

1. $\alpha \varepsilon<\varepsilon<(c-\phi) / 2$ and hence $q^{\mathrm{NE}}<1$ :

The gap in the average payoff for Sanchos between the asymptotically stable equilibrium and the Nash equilibrium reads:

$$
\bar{\pi}^{\mathrm{S}}\left(0, q_{\mathrm{Q}}^{*}\right)-\bar{\pi}^{\mathrm{S}}\left(0, q^{\mathrm{NE}}\right)=\frac{1}{2}(c-d+\phi)\left(q_{\mathrm{Q}}^{*}-q^{\mathrm{NE}}\right)<0 .
$$

2. $\alpha \varepsilon<(c-\phi) / 2<\varepsilon$ and hence $q^{\mathrm{NE}}=1$ :

The gap in the average payoff for Sanchos between the asymptotically stable equilibrium and the Nash equilibrium reads:

$$
\bar{\pi}^{\mathrm{S}}\left(0, q_{\mathrm{Q}}^{*}\right)-\bar{\pi}^{\mathrm{S}}(0,1)=-\frac{1}{2} \sigma\left(1-q_{\mathrm{Q}}^{*}\right)<0
$$

Acknowledgements We would like to thank Guiomar Martín-Herrán and Martin Kesternich for useful comments and suggestions. We are also grateful to the two anonymous referees. The final version of this article has greatly benefited from their critics, comments and remarks.

\section{Compliance with Ethical Standards:}

Funding: This study was funded by the Spanish Government (projects ECO201452343-P and ECO2017-82227-P), as well as financial aid from Junta de Castilla y León (projects VA024P17 and VA105G18), co-financed by FEDER funds.

Conflict of Interest: The authors declare that they have no conflict of interest.

\section{References}

1. Antoci A, Borghesi S, Russu P (2012) Environmental protection mechanisms and technological dynamics. Econ Model 29(3):840-847. doi:10.1016/j.econmod.2011.10.004

2. Antoci A, Galeotti M, Radi D (2011) Financial tools for the abatement of traffic congestion: A dynamical analysis. Comput Econ 38:389-405. doi:10.1007/s10614-011-9294-7

3. Antoci A, Dei R, Galeotti M (2009) Financing the adoption of environment preserving technologies via innovative financial instruments: An evolutionary game approach. Nonlinear Anal 71:952-959. doi: 10.1016/j.na.2009.01.077

4. Dawes RM (1975) Formal models of dilemmas in social decision making. In: Kaplan M F, Schwartz S (eds) Human Judgement and Decision Making. New York Academic, 87-107 
5. Doebeli M, Hauert C, Killingback T (2004) The Evolutionary Origin of Cooperators and Defectors. Science 5697: 859-862. doi: 10.1126/science.1101456

6. Liebrand WBG (1983) A classification of social dilemma games. Simulat Gaming 14(2): 123-138. doi: https://doi.org/10.1177/104687818301400201

7. Mailath GJ (1998) Do people play Nash equilibrium? Lessons from evolutionary game theory. J Econ Lit 36(3):1347-1374. doi:10.1016/0165-1765(91)90047-O

8. Oprea R, Henwood K, Friedman D (2011) Separating the Hawks from the Doves: Evidence from continuous time laboratory games. J Econ Theory 146(6):2206-2225. doi:10.1016/j.jet.2011.10.014

9. Samuelson L, Zhang J (1992) Evolutionary stability in asymmetric games. J. Econ. Theory 57:363-391. https://doi.org/10.1016/0022-0531(92)90041-F

10. Sandholm WH (2010) Population Games and Evolutionary Dynamics. MIT Press, Cambridge

11. Selten R (1980) A note on evolutionarily stable strategies in asymmetric animal conflicts. J Theoret Biol 84(1):93-101. https://doi.org/10.1016/S0022-5193(80)81038-1 